\title{
The Urban Emergency Managing System Based on the Internet of Things
}

\author{
Guowei.Yu \\ Shenyang Ligong University \\ NO 6 NanPing Road,Shenyang City \\ Liaoning Province,China \\ e-mail: ygwhome@sina.com
}

\author{
Jingyu.Wang \\ Shenyang Ligong University \\ NO 6 NanPing Road,Shenyang City \\ Liaoning Province,China \\ e-mail: wangjy888@163.com
}

\begin{abstract}
The theories of emergency managing have been studied widely. However, the researches concerning the introduction of the Internet of things are scarce. A general solution plan of the urban emergency managing system based on the Internet of things is proposed. The emergency managing system involves four categories, which are perception layer, network layer, business layer and interface layer. Through the technology of the Internet of things, the carrying-out ability and decision-making intelligence are enhanced greatly. The four-layer framework is helpful for the construction of an open, flexible and general integrated information system in the course of emergency managing.
\end{abstract}

Keywords-Internet of things;Emergency managing;Managing information system;Four-layer structure

\section{INTRODUCTION}

The emergency system is a system with complicated knowledge, which is related to electronic information engineering, management, coordination, network communication and many other disciplines[1]. The urban emergency system should be combined with some advanced theories and the knowledge of some related disciplines so as to reach the optimal stage in the face of emergencies[2,3,4,5]. The optimal stage involves the optimal planning of the emergency planning, the optimizing of decision-making, the effective controlling and managing. With the help of the advanced computer techniques and information techniques, DSS and EMS can deal with the increasingly complicated daily problems successfully. The existing EMS can be categorized into three groups, which are EMS based on gis techniques[6], expert EMS and EMS based on multiintellectualities. According to the analysis of the practical needs, we conclude that the urban emergency managing system built upon the Internet of things has a promising prospective.

Emergency managing system usually includes six sub-systems, which are the monitoring system of emergencies in the field of public health (sensing), the data transmitting and collecting system (network), the supporting system of emergency decision-making (thinking), the emergency coordinating system (coordination), the managing system of emergency administration (flexibility) and the resources managing system. If the boundaries of different sub-systems are not clarified, the problems of repetitive development, excessive data, lengthened development cycle and excessive work will emerge due to the individual perspective of the developers[7]. Emergency managing system has become an important component of the development of electronic administration. It is also indispensible to the functioning of modern digitalized cities. The information of public facilities and various enterprises have become more and more important for the administrative departments' emergency decision-making. The information of enterprises is usually integrated into ERP, HR systems and E-business systems[8]. The separate information as to public facilities is scattered into various fields. Thus, it is beneficial and applicable to build a supporting system upon the Internet of things for the urban emergency decision-making. In this way, various resources can be integrated into one emergency managing system. The techniques of Internet of things can increase the depth of the controlling of the emergency managing platform so that the managing is more intellectual and convenient with widened space and immediate functions.

Based on the existing research and practice, we can conclude that the results of emergency managing systems in cities can be optimized when they are built upon the existing information system and the practical needs of the work. They should be combined with the existing public facilities and network telecommunication facilities. With the development of general interface, the integration and intellectualization of emergency management can be realized. In this article, an EMS framework based on the Internet of things is put forward. The application of this framework is significant in shortening the developing cycle, reducing the cost and facilitating the using and maintaining. Its design is combined with the modern controlling techniques, modern telecommunication techniques, modern optimized coordinating techniques and distributed software techniques. The framework is composed of emergency monitoring system, emergency decision-making supporting system, the emergency commanding and coordinating system, emergency administrating system and resources managing system. Its function includes instant monitoring, early warning, starting, handling and post-handling. We will set up an integrated platform of emergency managing based on SOA framework with the help of Internet of things techniques, EAI techniques and telecommunication techniques. Firstly, through this emergency managing framework, the development of EMS system can be based on the existing infrastructures so as to greatly reduce the work of developing and maintaining the system. Secondly, when emergency occurs, the coordination of EMS system and other systems can be realized timely. Thirdly, EMS 
system should have flexible structures so as to avoid the emergence of data isolated islands and reducing the maintaining work.

\section{THE SOLUTION OF EMS}

The solution of EMS for the urban emergencies in the system is shown in Fig.1. Vertically, it is composed of four layers, which are data perception layer, network communication layer, business application layer and the interface layer for integration service. Business application layer can be sub-divided into three layers, which are the presentation layer, the business logic layer and the data storage layer. The features of various layers will be described in the following parts.

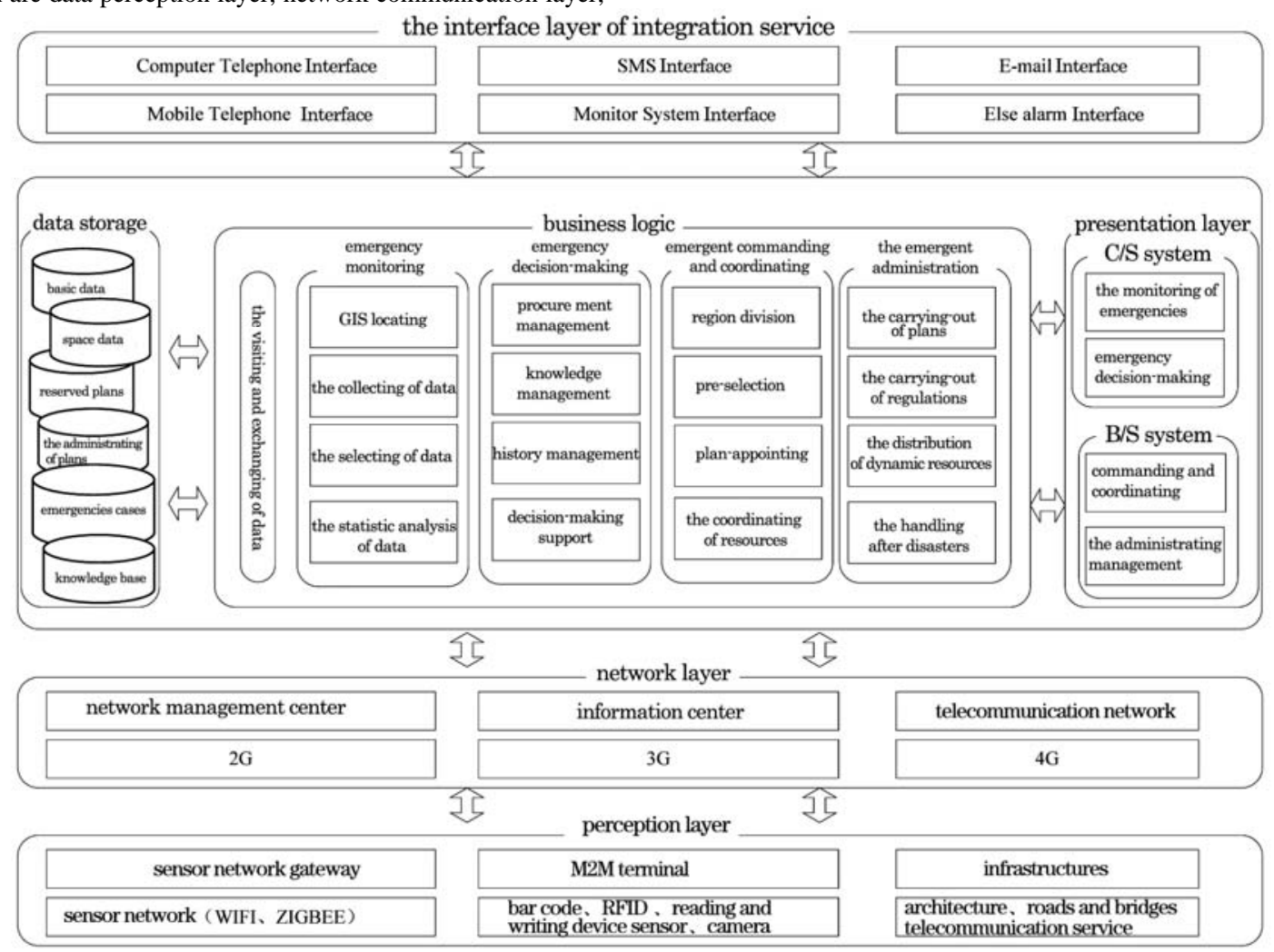

Fig .1 the framework chart for the emergency information managing system based on the Internet of things

A.

\section{Data perception layer}

This layer consists of various sensors as well as sensor gateways. It is responsible for monitoring the condition and collecting data for public environment (including the concentration of $\mathrm{CO} 2$, the moisture and the temperature), public facilities (such as the signs of roads and bridges, architectures and the telecommunication benchmarking) and other facilities. The facilities of perception layer include bar codes, RFID labels, writing and reading devices, cameras, GPS positioning instruments, and perception terminals such as wireless sensor network. This layer serves as the sensing endings or the sensing organs such as eyes, ears, nose and tongue. It is the physical layer for the Internet of things to recognize objects and collect information. Through combining various sensing methods and data-collecting methods, this layer builds up the capacity of sensing the surroundings in various aspects and managing and controlling emergencies comprehensively on the emergency platforms.

\section{B. Network communication layer}

This layer is the nerve center of the emergency management platform. It is responsible for the transmission and integration of the concerning information. It consists of the internet, the network management system, intelligent processing center (the selecting, distributing and forwarding of data), data center (the storage of data), etc. It combines the computer telecommunication integration techniques (CTI), the satellite positioning system (GPS), the spatial information systems (GIS) together. The combination of the existing network telecommunication infrastructure, the telephone paging system and GPS, the early warning, spatial positioning and visualization display of the emergencies can be realized. The specific functions this layer can perform include: 1) the autonomous noting of the alarm phone calls; 2) the audio coordination based upon CTI; 3) the interactive display and consultation between the alarming information and the electronic map; 4) the autonomous informing of the emergencies the information-collecting of the perception layer, the analyzing and selecting of information and the 
transmitting of information to the remote commanding system; 5) the dynamic monitoring of the condition of the emergencies, the reflection of the position and seriousness of the emergencies. In order to achieve the remote coordinating and commanding effectively, the staff members can apply the following methods: 1) the coordinating base on voice calls, cable network and IP telephones; 2) the wireless coordination through intensive broadcasting, portable radios, wireless paging systems, mobile phone systems, wireless base stations and satellite telecommunication systems.

\section{Business application layer}

This layer is responsible for providing the interface between the emergency managing system and the users (including individuals, organizations and other systems). It meets the needs of the emergency managing. With its help, the society and the corresponding organizations can manage various emergencies effectively. This layer is constructed upon the typical $\mathrm{B} / \mathrm{S}$ framework. It belongs to the upper layer of the emergency managing system. It can be sub-divided into three layers, which are the presentation layer, the business logic layer and the data storage layer.

\section{1)Presentation layer}

This layer is characterized by the appearance and layout of the users' interface. The interface of emergency managing system should realize the combination of $\mathrm{C} / \mathrm{S}$ style with $\mathrm{B} / \mathrm{S}$ style in order to reinforce the displaying capacity. Since the emergency managing system and the monitoring system are related to a lot of basic data displayed in the hierarchical tree structure, the C/S style is preferable. On the other hand, the emergency commanding and coordinating system as well as the emergency administrating system are responsible for commanding the whole action and taking specific measures. They will handle various emergencies and deal with various systems and staff members. Thus, B/S style is more suitable. The presentation layer is designed and developed according to MVC model. The concerning techniques include Java Servlet and JSP. The final aim is to provide the data-presenting ability with multiple configurations.

\section{2)Business logic layer}

The business logic layer is the central step for the application layer of the emergency managing system. According to the various functions, the emergency managing platform structure can be divided into four modules, which are the sub-system of monitoring emergencies, the sub-system of decision-making supporting, the sub-system of commanding and coordinating, the sub-system of handling emergencies. The sub-system of monitoring emergencies is responsible for collecting and monitoring data, transmitting sensitive data and locating alarming information. The sub-system of decision-making supporting is responsible for managing emergency-handling plans and knowledge, managing the emergency-handling records and supporting decision-making. The sub-system of commanding and coordinating is able to recognize and define various emergencies, choose the emergency-handling model and design emergency-handling plans promptly. Then, the specific measures and solutions can be obtained on the basis of the situation of material and human resources. The sub-system of handling emergencies is capable of providing the supporting, administrating and monitoring function for carrying out emergency-handling plans.

EJB techniques are used in the business logic layer. The EJB technique definitions are completed by the SUN Company. They are used to develop and deploy multi-layer structures as well as the Java component structures of the distributed and object-oriented platform. The bean of EJB is operated at the server side. It is the components of the server side based on the Java platform. It is used for the construction of the extendable software of the server sides. EJB includes two construction models, which are the dialogue bean and the entity bean. In order to respond to the requests of remote work, the EJB container can generate a dialogue bean, which declares an interoperation between the remote user and it. In the dialogue, the bean can coordinate and control the users' information. Meanwhile, the entity bean can provide a data view from the database. Many server sides can visit the same entity bean through the context at the same time. During this course, the basic data can be revised so that the completeness of the data can be guaranteed. Generally, the case-using models are used to define and realize the dialogue bean. The object models are used to define and realize the entity bean. In addition, the emergency system needs to use the various functions provided by the external business system so as to realize the provision of materials, the acquisition of resources and the deployment of human resources. A relationship of mutual exploitation can be established through businesses so as to complete the transmission of data information and realize various business logics. In this layer, the intellectual agents in the distributed environment can be exploited so that the business logics can be completed to enhance the intellectual level of the emergency managing platform.

\section{3) Data storage layer}

The data storage layer is used to store all the systematic data, including the basic resources, the space data, the planning data, the case data and knowledge base. These data can be stored in the relation database. The data-storing can be realized in documents of various formats, such as XML documents, office documents and txt documents.

This project is designed to exploit MS SQL database server to store relation data and manage business data. Meanwhile, SuperMap SDX space database server is exploited to manage the space data. Through the database server, the data can be stored, indexed, read and renewed. In addition, because the data-storing is realized in various document formats, document server needs to manage all the documents together. The framework is designed to use Microsoft IIS as the WEB server and document-managing server. The data in various forms are visited in various styles. The framework will add the data visiting and transforming service between the business logic layer and the data layer so as to realize the visiting of documents in various forms. Meanwhile, this layer can exchange data with the network layer and the integration service layer. 


\section{Integration service interface layer}

This layer provides other layers of the emergency managing platform with an integrated telecommunication interface service. Generally, after the emergencies occur, all the possible human resources and material resources should be deployed to deal with the urgent situation. In order to improve the automation level of the emergency managing and lessen the data contradiction after the occurrence of the emergencies, an integrated service strategy based on SOA framework is put forward in this project. It is used in the integration of the various functions of EMS and the interconnection between the emergency managing system and the external system. It contains the business logics of the emergency managing system so as to realize the services of monitoring emergencies, supporting decision-making, commanding and coordinating together with managing comprehensively. Meanwhile, a standardized interface with the essential functions of EMS for external users to visit is offered. In addition, during the operation of some emergency managing systems, some functions and data provided by the existing information system will be applied repeatedly. In order to guarantee the universality of emergency managing systems and the flexibility of version maintenance, a framework should be constructed so as to integrate the existing systems and meet the needs of mutual visiting between the business logic layers and the external systems while keeping the different technological standards. SOA is a component model with well-defined contract interface, which can connect different application units. It uses middleware technology to define a function interface which is independent of hardware platform, operating system and programming language. It uses a universal method to deploy the network service. SOA is not only a language but also a technique. What's more, it is a product. In its application, various methods are exploited according to the choices of development. At present, the concerning middle techniques include COM, CORBA and WEB SERVER. Due to the superior reliability and extendibility of WEB SERVER, it becomes the first technological choice to realize SOA. WEB SERVER is a distributed software application program labeled through URL. Its interface can be defined, described and searched through the message method or the Internet protocol. The structure includes the supplier of network service, the service requester, the service agents (telecommunication agents). The transmission of message data is fulfilled in XML format. WSDL, SOAP and UDDI are the integral techniques to realize WEB SERVER. WSDL (Web Services Description Language) is use to describe the standards of web services UDDI (Universal Description
Discovery and Integration) is used to distribute and search services. SOAP (Simple Object Access Protocol) is a protocol for the distributed lightweight calculation. The data formats and basic information are exchanged among the nodes of EMS through XML methods so that the distributed calculation is accomplished.

\section{CONCLUSION}

The construction, application and operation of emergency managing system involve a lot of work and information. An effective and efficient EMS should be established. The system can monitor each stage of the emergencies. It can also monitor the developing and handling of the emergencies timely. At the same time, the information can be collected and distributed so that the information can be transmitted in the overall system safely. The speed of dealing with the internal and external emergencies can be enhanced. The integrity and connectedness of the whole system can be improved. Under various emergent circumstances, measures are taken to avoid disasters or control the worsening or spreading of the disasters. Measures of relief can also be taken so that the reconstruction work after the disasters can be started at once. After completing the relief work, experience should be summarized timely so that the responding time of EMS can be improved. The accumulated knowledge can provide technological support for the future work. The emergency managing system can reduce the operating cost of handling emergencies. Meanwhile, it can shorten the operation cycle and improve the exactness of information-searching as well as the quality of emergency decision-making. The EMS plan lays a solid foundation for the establishment of the flexible emergency managing information system.

\section{REFERENCES}

[1] T Elbir: Atmospheric Environment, Vol 38(2004), p. 4509. (in Chinese)

[2] H X Lan, C H Zhou, L J Wang, et al: Engineering Geology, Vol. 76(2004), p.109. (in Chinese)

[3] A Zerger, D I Smith: Computers, Environment and Urban Systems, Vol. 27(2003), p.123. (in Chinese)

[4] P Avesan, A Perin, F Ricc: Applied Intelligence, Vol. 13(2000), p.41. (in Chinese)

[5] J ZHernández, JM Serrano: Expert Systems with Applications, Vol.20(2001), p.173. (in Chinese)

[6] S PKourniotis, C TKiranoudis, N CMarkatos: Safety Science, Vol. 38(2001), p.49. (in Chinese)

[7] J LWybo, K M Kowalsk: Safety Science, Vol.30(1998), p.131. (in Chinese)

[8] Kevin FR Liu: Expert Systems with Applications, Vol. 27(2004), p.77. (in Chinese) 